$\mathrm{UBC} / \mathrm{S}-95 / 1$

OUTP-95-46P

hep-th/9511199

June 28, 2021

\title{
Conformal Motions and the Duistermaat-Heckman Integration Formula
}

\author{
Lori D. Paniak and Gordon W. Semenoff \\ Department of Physics, University of British Columbia \\ Vancouver, British Columbia, Canada V6T $1 Z 1$ \\ Richard J. Szabo \\ Department of Theoretical Physics, University of Oxford \\ 1 Keble Road, Oxford OX1 3NP, U.K.
}

\begin{abstract}
We derive a geometric integration formula for the partition function of a classical dynamical system and use it to show that corrections to the WKB approximation vanish for any Hamiltonian which generates conformal motions of some Riemannian geometry on the phase space. This generalizes previous cases where the Hamiltonian was taken as an isometry generator. We show that this conformal symmetry is similar to the usual formulations of the Duistermaat-Heckman integration formula in terms of a supersymmetric Ward identity for the dynamical system. We present an explicit example of a localizable Hamiltonian system in this context and use it to demonstrate how the dynamics of such systems differ from previous examples of the Duistermaat-Heckman theorem.
\end{abstract}

\footnotetext{
${ }^{1}$ This work was supported in part by the Natural Sciences and Engineering Research Council of Canada.
} 
Understanding the circumstances under which a partition function is given exactly by its semi-classical approximation reveals connections between classical mechanics and the geometry and topology of the associated phase space. The Duistermaat-Heckman theorem [1] - [3] has been the basis of so-called localization theory in both mathematics and physics [4]-[12] (see [13] for a recent review) and it provides geometric criteria for the exactness of the semiclassical approximation for the finite-dimensional partition function

$$
Z(T)=\int_{\mathcal{M}} d^{2 n} x \sqrt{\operatorname{det} \omega(x)} \mathrm{e}^{i T H(x)}
$$

which describes the statistical dynamics (with imaginary temperature) of a classical Hamiltonian system. Here $\mathcal{M}$ is a $2 n$-dimensional phase space and $\omega_{\mu \nu}(x)$ is an antisymmetric tensor field on $\mathcal{M}$ which is non-degenerate, $\operatorname{det} \omega(x) \neq 0 \quad \forall x \in \mathcal{M}$, and whose matrix inverse $\omega^{\mu \nu}$ defines the Poisson brackets $\left\{x^{\mu}, x^{\nu}\right\}=\omega^{\mu \nu}(x)$ of the dynamical system. $H$ is a smooth Hamiltonian function on $\mathcal{M}$ which for simplicity we assume has a finite set of critical points $I(H)=\{p \in \mathcal{M}: d H(p)=0\}$ each of which is non-degenerate.

The partition function (1) has an asymptotic expansion for large- $T$ with coefficients determined by the method of stationary-phase approximation [14]. The Duistermaat-Heckman theorem states that if the phase space $\mathcal{M}$ is compact and closed and the classical timeevolution $x(t)$ of the dynamical system traces out a torus $\left(S^{1}\right)^{m}$ in $\mathcal{M}$, then the partition function (1) is given exactly by the leading order term

$$
Z^{(0)}(T)=\left(\frac{2 \pi}{T}\right)^{n} \sum_{p \in I(H)} \mathrm{e}^{i \frac{\pi}{4} \eta_{H}(p)} \sqrt{\frac{\operatorname{det} \omega(p)}{\operatorname{det} \mathcal{H}(p)}} \mathrm{e}^{i T H(p)}
$$

of its stationary-phase loop-expansion [1]. Here $\mathcal{H}(x)_{\mu \nu} \equiv \partial_{\mu} \partial_{\nu} H(x)$ is the non-degenerate Hessian matrix of $H$ and $\eta_{H}(p)$ is the spectral asymmetry of the Hessian at $p$ (the difference between the number of positive and negative eigenvalues of $\mathcal{H}(p)$ ).

A fundamental assumption that leads to the Duistermaat-Heckman theorem and its generalizations [3] is the existence of a globally-defined metric tensor $g=\frac{1}{2} g_{\mu \nu}(x) d x^{\mu} d x^{\nu}$ on $\mathcal{M}$ which is invariant under the classical flows $x(t) \in \mathcal{M}$ of the Hamiltonian system, i.e.

$$
g(x(t))=g(x(0))
$$

The condition (3) is a very restrictive one on the Hamiltonian dynamics as it implies that $H$ must generate a global $U(1)$-action on $\mathcal{M}[10]$. The set of Hamiltonian systems which obey these constraints has been examined in [9, 11, 12]. In this Letter we will show that the Duistermaat-Heckman integration formula (2) for the partition function (1) still holds when the geometric assumption (3) is replaced by the weaker condition that there exists a metric tensor $g$ on $\mathcal{M}$ which is invariant under the classical time evolution of the dynamical system up to a change of scale,

$$
g(x(t))=\mathrm{e}^{t \Lambda(x(t))} g(x(0))
$$


for some smooth function $\Lambda(x)$ on $\mathcal{M}$. We shall argue that this extended geometric requirement is similar to the isometry condition (3) from the point of view of localizing (1) onto the critical point set $I(H)$, except that the classical dynamics now possess behaviour not normally observed for systems whose partition functions can be localized. We illustrate these features for some explicit examples which show how the extension (4) expands the set of previously studied Hamiltonian systems for which the Duistermaat-Heckman formula holds.

First, we will discuss some features of the integration in (11). We describe the exterior differential calculus of the manifold $\mathcal{M}$ by introducing a set of anticommuting Grassmann variables $\psi^{\mu}$ which are to be identified locally with the basis elements $\psi^{\mu} \sim d x^{\mu}$ of the cotangent bundle $T^{*} \mathcal{M}$ of $\mathcal{M}$. A differential $m$-form is represented by contracting a rank- $m$ antisymmetric tensor function on $\mathcal{M}$ with $\psi^{\mu_{1}} \cdots \psi^{\mu_{m}}$ and it can be regarded as a function $\eta(x, \psi)=\frac{1}{m !} \eta_{\mu_{1} \cdots \mu_{m}}(x) \psi^{\mu_{1}} \cdots \psi^{\mu_{m}}$ on the super-manifold $\mathcal{M} \otimes T^{*} \mathcal{M}$. The integration of differential forms is defined on $\mathcal{M} \otimes T^{*} \mathcal{M}$ by introducing the usual Berezin rules for integrating Grassmann variables, $\int d \psi^{\mu} \psi^{\mu}=1, \int d \psi^{\mu} 1=0$. With these rules, we can absorb the determinant of the symplectic 2 -form $\omega \equiv \frac{1}{2} \omega_{\mu \nu}(x) \psi^{\mu} \psi^{\nu}$ into the exponential in (1) and write the classical partition function as

$$
Z(T)=\frac{1}{(i T)^{n}} \int_{\mathcal{M} \otimes T^{*} \mathcal{M}} d^{2 n} x d^{2 n} \psi \quad \mathrm{e}^{i T S(x, \psi)} \equiv \int_{\mathcal{M}} \alpha
$$

where we have introduced the inhomogeneous differential form

$$
\alpha=\frac{1}{(i T)^{n}} \mathrm{e}^{i T(H+\omega)}=\mathrm{e}^{i T H} \sum_{k=0}^{n} \frac{(i T)^{k-n}}{k !} \omega^{k}
$$

From the Berezin rules the integration in (5) is non-zero only on the top-form (degree $2 n$ ) component $\omega^{n}$ of $\alpha$, and all forms in (6) of Grassmann-degree higher than $2 n$ vanish because of the fermionic nature of the variables $\psi^{\mu}$. The integral in (5) can be thought of as the partition function of a zero-dimensional quantum field theory with bosonic fields $x^{\mu}$, fermion fields $\psi^{\mu}$, and action $S(x, \psi) \equiv H(x)+\omega(x, \psi)$.

The Hamiltonian vector field is defined by the equation

$$
V^{\mu}(x) \equiv \omega^{\mu \nu}(x) \partial_{\nu} H(x) \quad \text { or } \quad d H=-i_{V} \omega
$$

where the exterior derivative operator $d=\psi^{\mu} \frac{\partial}{\partial x^{\mu}}$ maps $m$-forms to $(m+1)$-forms, and $i_{V}=$ $V^{\mu}(x) \frac{\partial}{\partial \psi^{\mu}}$ is the interior multiplication operator which contracts differential forms to 1 lower degree with the vector field $V$. Both $d$ and $i_{V}$ are nilpotent, $d^{2}=\left(i_{V}\right)^{2}=0$, and are graded derivations, i.e. they define operators $\mathcal{Q}$ whose action on differential forms obeys the graded Leibniz rule $\mathcal{Q}(\eta \beta)=(\mathcal{Q} \eta) \beta+(-1)^{m} \eta(\mathcal{Q} \beta)$, where $\eta$ is an $m$-form. The flows

$$
\dot{x}^{\mu}(t)=V^{\mu}(x(t))
$$


of the Hamiltonian vector field define the classical equations of motion of the dynamical system. Note that by definition the symplectic 2 -form $\omega$ is closed, i.e. $d \omega=0$.

We now introduce the Cartan equivariant exterior derivative operator [3]

$$
Q_{V}=d+i_{V}
$$

which is a graded derivation that maps $m$-forms into the sum of $(m-1)$ - and $(m+1)$-forms. If we think of commuting, even-degree forms as representing bosons and anti-commuting, odd-degree forms as representing fermions, then this suggests that $Q_{V}$ represents some sort of supersymmetry operator in the dynamical theory. However, unlike the operators $d$ and $i_{V}$, $Q_{V}$ is not nilpotent in general. The square of $Q_{V}$ is given by the Weil identity

$$
Q_{V}^{2} \beta=\left(d i_{V}+i_{V} d\right) \beta=\mathcal{L}_{V} \beta
$$

for the Lie derivative $\mathcal{L}_{V}$ along $V$ acting on differential forms. The form $\mathcal{L}_{V} \beta$ represents the infinitesimal $(t \rightarrow 0)$ variation as $x(0) \rightarrow x(t)$ of the form $\beta$ under the flows (8) of $V$. The operator $Q_{V}$ is therefore nilpotent on the kernel ker $\mathcal{L}_{V}=\left\{\beta \in \Lambda^{*} \mathcal{M}: \mathcal{L}_{V} \beta=0\right\}$ of the linear derivation $\mathcal{L}_{V}$ which represents the subspace of differential forms which are invariant under the classical dynamics of the Hamiltonian system, i.e. for which $\beta(x(t))=\beta(x(0))$. Such forms are known as equivariant differential forms [3].

From the definition (7) and the fact that $\omega$ is closed it follows that the action in (5) satisfies

$$
Q_{V} S(x, \psi)=\left(d+i_{V}\right)(H+\omega)=d H+i_{V} \omega \equiv 0
$$

This means that, if we interpret $Q_{V}$ as a supersymmetry charge, then the action $S$ is supersymmetric and the partition function (5) determines a supersymmetric quantum field theory. In this setting, $Q_{V}$ determines an $N=\frac{1}{2}$ supersymmetry algebra $Q_{V}^{2}=\mathcal{L}_{V}$ and the BRST complex of physical states is the space $\operatorname{ker} \mathcal{L}_{V}$ of equivariant differential forms. In the mathematics literature the BRST cohomology of the charge $Q_{V}$ (i.e. the space of $Q_{V}$-closed forms $\eta, Q_{V} \eta=0$, modulo $Q_{V}$-exact forms $\left.\eta=Q_{V} \beta\right)$ is called the $U(1)$-equivariant cohomology of $\mathcal{M}$ generated by the action of $V$ on $\mathcal{M}$ 22, 3, 13. Notice also that, because of the Leibniz rule for $Q_{V}$, the differential form (6) is also supersymmetric, $Q_{V} \alpha=0$.

We shall now derive a general integration formula for the partition function (1) in terms of geometrical objects on the phase space which will allow us to examine its localization features explicitly. Consider the integral

$$
\mathcal{Z}(s)=\int_{\mathcal{M}} \alpha \mathrm{e}^{-s Q_{V} \beta}
$$

where $\beta$ is an arbitrary globally defined differential form on $\mathcal{M}$. We assume that $\mathcal{Z}(s)$ is a regular function of $s \in \mathbb{R}^{+}$and that its $s \rightarrow 0$ and $s \rightarrow \infty$ limits exist. Its $s \rightarrow 0$ limit is just 
the integral $Z(T)=\int_{\mathcal{M}} \alpha$ of interest while its $s \rightarrow \infty$ limit represents a localization of (5) onto the smaller subspace of $\mathcal{M}$ where $Q_{V} \beta=0$. Then (12) and the identity

$$
\mathcal{Z}(0)=\lim _{s \rightarrow \infty} \mathcal{Z}(s)-\int_{0}^{\infty} d s \frac{d}{d s} \mathcal{Z}(s)
$$

imply that the partition function (5) can be determined as

$$
Z(T)=\lim _{s \rightarrow \infty} \int_{\mathcal{M}} \alpha \mathrm{e}^{-s Q_{V} \beta}+\int_{0}^{\infty} d s \int_{\mathcal{M}} Q_{V}(\alpha \beta) \mathrm{e}^{-s Q_{V} \beta}
$$

where we have used the fact that $\alpha$ is supersymmetric.

Consider first the last integration in (14). Since $Q_{V}$ is a graded derivation we can integrate by parts to get

$$
\int_{\mathcal{M}} Q_{V}(\alpha \beta) \mathrm{e}^{-s Q_{V} \beta}=\int_{\mathcal{M}} Q_{V}\left(\alpha \beta \mathrm{e}^{-s Q_{V} \beta}\right)+\int_{\mathcal{M}} \alpha \beta Q_{V}\left(\mathrm{e}^{-s Q_{V} \beta}\right)
$$

In the first integral on the right-hand side of (15) there is an $i_{V}$-exact integrand. Since interior multiplication reduces the order of a form by one and integration over the manifold is nonzero only on the top-degree component of any differential form, it follows that this integral vanishes. As for the $d$-exact integration in this same integral, we can use Stokes' theorem to write it as an integral over the $(2 n-1)$-dimensional boundary $\partial \mathcal{M}$ of the manifold $\mathcal{M}$. Finally, in the last integral we recognize the Lie derivative from (10), and hence

$$
\int_{0}^{\infty} d s \int_{\mathcal{M}} Q_{V}(\alpha \beta) \mathrm{e}^{-s Q_{V} \beta}=\int_{0}^{\infty} d s\left\{\oint_{\partial \mathcal{M}} \alpha \beta \mathrm{e}^{-s Q_{V} \beta}-s \int_{\mathcal{M}} \alpha \beta\left(\mathcal{L}_{V} \beta\right) \mathrm{e}^{-s Q_{V} \beta}\right\}
$$

To carry out the first integration in (14), we must explicitly specify the form $\beta$. In principle there are many possibilities for $\beta$ [10, 11, 13], but in order to obtain finite results in the limit $s \rightarrow \infty$ we need to ensure that the form $Q_{V} \beta$ has a 0 -form component to produce an exponential damping factor, since higher order forms will contribute only polynomially due to antisymmetry (see (6)). This is guaranteed only if $\beta$ has a 1-form component. Thus it is only the 1 -form part of $\beta$ that will be relevant in the following, and so without loss of generality we assume that $\beta \equiv B_{\mu} \psi^{\mu}$. Furthermore, we need for the 0 -form part $V^{\mu} B_{\mu}$ of $Q_{V} \beta$ to attain its global minimum at zero so that the large-s limit of (14) yields a non-zero result. This boundedness requirement is equivalent to the condition that the component of $B$ along $V$ has the same orientation as $V$. In order to implement such a condition we need to introduce a globally defined Euclidean-signature metric tensor $g_{\mu \nu}(x)$ on the phase space. Then the most general form of $\beta$ up to components orthogonal to $V$ is given by

$$
\beta(x, \psi)=f(x) g(V, \cdot) \equiv f(x) g_{\mu \nu}(x) V^{\mu}(x) \psi^{\nu}
$$

where $f(x)$ is any strictly-positive smooth-function on $\mathcal{M}$. 
With this choice for $\beta$ we have $Q_{V} \beta=K_{V}+\Omega_{V}$ where

$$
K_{V}=f \cdot g(V, V) \equiv f \cdot g_{\mu \nu} V^{\mu} V^{\nu} \quad, \quad \Omega_{V}=d[f \cdot g(V, \cdot)] \equiv f\left(2 g \cdot \nabla V-\mathcal{L}_{V} g\right)+(d f) g(V, \cdot)
$$

Here $\nabla \equiv d+\Gamma$ is the usual covariant derivative with $\Gamma$ the Levi-Civita-Christoffel (affine) connection associated with the Riemannian metric $g$, and

$$
\left(\mathcal{L}_{V} g\right)_{\mu \nu}=g_{\mu \lambda} \nabla_{\nu} V^{\lambda}+g_{\nu \lambda} \nabla_{\mu} V^{\lambda}
$$

are the components of the Lie derivative of $g$ along $V$. We now substitute these identities into (14) to write the first integration there as a sum over the critical point set $I(H)$ which coincides with the zero locus of the Hamiltonian vector field $V$,

$$
\begin{aligned}
\lim _{s \rightarrow \infty} \int_{\mathcal{M}} \alpha \mathrm{e}^{-s Q_{V} \beta}= & \lim _{s \rightarrow \infty} \int_{\mathcal{M} \otimes T^{*} \mathcal{M}} d^{2 n} x d^{2 n} \psi \frac{\mathrm{e}^{i T\left(H+\frac{1}{2} \omega_{\mu \nu} \psi^{\mu} \psi^{\nu}\right)}}{(i T)^{n}} \mathrm{e}^{-s f \cdot g_{\mu \nu} V^{\mu} V^{\nu}-\frac{s}{2}\left(\Omega_{V}\right)_{\mu \nu} \psi^{\mu} \psi^{\nu}} \\
= & \left(\frac{2 \pi i}{T}\right)^{n} \int_{\mathcal{M} \otimes T^{*} \mathcal{M}} d^{2 n} x d^{2 n} \psi \mathrm{e}^{i T\left(H(x)+\frac{1}{2} \omega_{\mu \nu}(x) \psi^{\mu} \psi^{\nu}\right)} f^{-n}(x) \frac{\delta(V(x))}{\sqrt{\operatorname{det} g(x)}} \\
\times & \operatorname{Pfaff} \Omega_{V}(x) \delta(\psi) \\
= & \left(\frac{2 \pi i}{T}\right)^{n} \sum_{p \in I(H)} \frac{f^{-n}(p) \mathrm{e}^{i T H(p)}}{|\operatorname{det} d V(p)|} \frac{\operatorname{Pfaff} \Omega_{V}(p)}{\sqrt{\operatorname{det} g(p)}}
\end{aligned}
$$

Finally, we can rewrite the determinants in (20) using the fact that at a critical point $p \in I(H)$ the Hessian of $H$ can be written using the Hamilton equations (0) as

$$
\mathcal{H}(p)_{\mu \nu} \equiv \partial_{\mu} \partial_{\nu} H(p)=-\left(\partial_{\mu} V^{\lambda}\right)(p) \omega_{\lambda \nu}(p)
$$

and likewise from the definition of the 2 -form $\Omega_{V}$ in (18) we have

$$
g^{\mu \lambda}(p)\left(\Omega_{V}\right)_{\lambda \nu}(p)=f(p)\left\{2 \omega^{\mu \lambda}(p) \mathcal{H}(p)_{\lambda \nu}-g^{\mu \lambda}(p)\left(\mathcal{L}_{V} g\right)_{\lambda \nu}(p)\right\}
$$

where $g^{\mu \nu}$ is the matrix inverse of $g_{\mu \nu}$. Substituting (21) and (22) into the large-s limit integral (20) in (14) and combining this with (16) and the choice of $\beta$ in (17), we arrive at our final expression for the integral (11) in terms of geometrical characteristics of the phase space

$$
\begin{aligned}
Z(T)= & \left(\frac{2 \pi}{T}\right)^{n} \sum_{p \in I(H)} \mathrm{e}^{i \frac{\pi}{4} \eta_{H}(p)} \sqrt{\frac{\operatorname{det} \omega(p)}{\operatorname{det} \mathcal{H}(p)}} \mathrm{e}^{i T H(p)} \sqrt{\operatorname{det}\left(\mathbf{1}-\mathcal{H}^{-1} \omega g^{-1} \mathcal{L}_{V} g / 2\right)(p)} \\
& +\frac{1}{(i T)^{n}} \int_{0}^{\infty} d s \oint_{\partial \mathcal{M}} \frac{\mathrm{e}^{i T H-s K_{V}}}{(n-1) !} f \cdot g(V, \cdot)\left(i T \omega-s \Omega_{V}\right)^{n-1} \\
& -\frac{1}{(i T)^{n}} \int_{0}^{\infty} d s s \int_{\mathcal{M}} \frac{\mathrm{e}^{i T H-s K_{V}}}{(n-1) !} f^{2} \cdot g(V, \cdot)\left(\mathcal{L}_{V} g\right)(V, \cdot)\left(i T \omega-s \Omega_{V}\right)^{n-1}
\end{aligned}
$$


where we have used the fact that the fermion field $g(V, \cdot)$ is nilpotent, and the factor $\mathrm{e}^{i \frac{\pi}{4} \eta_{H}(p)}$ takes into proper account of the sign of the Pfaffian Pfaff $d V(p) \sim \sqrt{\operatorname{det} \omega^{-1} \mathcal{H}(p)}$ in $(20)$ at each critical point $p \in I(H)$.

The expression (23) represents an alternative to the conventional loop-expansion [14 which explicitly takes into account the geometric symmetries that make the 1-loop approximation exact. It is readily seen that, for closed phases spaces $(\partial \mathcal{M}=\emptyset)$, (23) reduces to the Duistermaat-Heckman integration formula (2) whenever the Hamiltonian vector field $V$ is a conformal Killing vector of a metric $g$, i.e.

$$
\mathcal{L}_{V} g=\Lambda g
$$

which in local coordinates reads

$$
g_{\mu \lambda} \nabla_{\nu} V^{\lambda}+g_{\nu \lambda} \nabla_{\mu} V^{\lambda}=\frac{1}{n}\left(\nabla_{\lambda} V^{\lambda}\right) g_{\mu \nu}=\frac{1}{n}\left(\nabla_{\lambda} \omega^{\lambda \rho} \partial_{\rho} H\right) g_{\mu \nu}
$$

where the smooth-function $\Lambda(x)=\operatorname{tr} \nabla V(x) / n$ is fixed by contracting both sides of (24) with $g^{\mu \nu}$.

Notice that the scaling function $\Lambda(x)$ in (24) vanishes at the critical points of $H$, so that the only possible global Hamiltonian conformal Killing vectors are those which generate global isometries of $g, \Lambda(x) \equiv 0$ almost everywhere on $\mathcal{M}$, or non-homothetic transformations for which $\Lambda(x)$ is a globally-defined non-constant function on $\mathcal{M}$. The former case, wherein the Hamiltonian vector field is a Killing vector of some globally-defined Riemannian metric tensor on $\mathcal{M}$, is well-known to represent a quite general class of dynamical systems for which the Duistermaat-Heckman formula is exact [3], [5]-[13]. In that case, we set $f=1$ in (17) so that then $\mathcal{L}_{V} \beta=\left(\mathcal{L}_{V} g\right)(V, \cdot)=0$, i.e. $\beta \in \operatorname{ker} \mathcal{L}_{V}$ is a supersymmetric fermion field. Then the derivation of (23) also serves to show that the localization integral (12) coincides with the partition function (1) for all $s$. This is because the supersymmetric action $S-s Q_{V} \beta$ in $\mathcal{Z}(s)$ is cohomologous under $Q_{V}$ to the supersymmetric action $S$, and the partition function (5) depends only on the BRST cohomology class of $S$, and not on its particular representative. This feature is often refered to as the equivariant localization principle [2, 8, 9, 11, 13] - we can topologically renormalize the action $S(x, \psi)$ without changing the value of the integral $Z(T)$ in (5). This can be thought of as a Ward identity associated with the "hidden" supersymmetry of the dynamical theory. Notice that if $H$ has no stationary points on the boundary $\partial \mathcal{M}$ of $\mathcal{M}$, then the first $s$-integration on the right-hand side of (16) can be carried out explicitly and yields

$$
Z_{\partial \mathcal{M}}^{(0)}(T)=\oint_{\partial \mathcal{M}} \frac{\mathrm{e}^{i T H}}{g(V, V)} g(V, \cdot) \sum_{k=0}^{n-1} \frac{(-1)^{k}}{(n-k-1) !}\left(\frac{\Omega_{V}}{K_{V}}\right)^{k} \frac{\omega^{n-k-1}}{(i T)^{k+1}}
$$

which represents the additional contribution to the Duistermaat-Heckman integration formula (2) for manifolds with boundary [3]. 
The case of a non-vanishing $\Lambda(x)$ is similar to the isometry case from the point of view of the localization mechanism discussed above. Note that away from the critical points of $H$ we can choose the function $f(x)$ in (17) so that $\beta=g(V, \cdot) / g(V, V)$. With this choice for the localization 1-form $\beta$ it is easy to show that away from the critical point set of the Hamiltonian it satisfies $\mathcal{L}_{V} \beta=0$. Thus away from the subset $I(H) \subset \mathcal{M}$ the conformal Killing condition can be cast into the same supersymmetric context as the isometry condition by a rescaling of the metric tensor in (24), $g_{\mu \nu} \rightarrow G_{\mu \nu}=g_{\mu \nu} / g(V, V)$, for which $\mathcal{L}_{V} G=0$. Of course the rescaled metric $G_{\mu \nu}(x)$ is only defined on $\mathcal{M}-I(H)$, but all that is needed to establish the localization of (5) onto the zeroes of the vector field $V$ (i.e. the equivariant localization principle) is an invariant metric tensor (or equivalently an equivariant differential form $\beta$ ) which is defined everywhere on $\mathcal{M}$ except possibly in an arbitrarily small neighbourhood of $I(H)$ [7, 13].

However, in contrast to isometry generators the generators of conformal transformations need not correspond to a global $U(1)$-action on $\mathcal{M}$. This is because although the isometry group of a compact space is itself compact, the conformal group need not be. We might therefore expect that globally the case of a non-vanishing scaling function $\Lambda(x)$ in (24) represents a new sort of localizable Hamiltonian dynamics. To explore this possibility, we now turn to an explicit example of a Hamiltonian system which generates non-zero conformal motions of a Riemannian metric. We consider the plane $\mathcal{M}=\mathbb{R}^{2}$ with its usual flat Euclidean metric which in complex coordinates is $d s^{2}=d z d \bar{z}$. In this case the conformal Killing equations (25) become simply $\partial_{\bar{z}} V^{z}=\partial_{z} V^{\bar{z}}=0$, and thus the conformal group of the flat plane is generated by arbitrary holomorphic vector fields $V^{z}=F(z), V^{\bar{z}}=\bar{F}(\bar{z})$ (these generate the infinite-dimensional classical Virasoro algebra). The classical equations of motion determined by the Hamiltonian flows of these vector fields are therefore the arbitrary analytic coordinate transformations

$$
\dot{z}(t)=F(z(t)) \quad, \quad \dot{\bar{z}}(t)=\bar{F}(\bar{z}(t))
$$

We shall now explicitly construct a Hamiltonian system associated with such a vector field. For definiteness, we consider the conformal Killing vector which describes a Hamiltonian system with $n+1$ distinct stationary points,

$$
V^{z}=i \beta z\left(1-\alpha_{1} z\right) \cdots\left(1-\alpha_{n} z\right)
$$

at $z=0$ and $z=1 / \alpha_{i}$, where $\beta, \alpha_{i} \in \mathbb{C}$. The associated scaling function in (24) is then

$$
\Lambda(z, \bar{z})=\partial_{z} V^{z}+\partial_{\bar{z}} V^{\bar{z}}
$$

The integrability of the Hamiltonian equations (17) requires that the symplectic 2-form be invariant under the flows of $V$, i.e. $\mathcal{L}_{V} \omega=d i_{V} \omega=0$. This leads to the first-order linear 
partial differential equation

$$
V^{z} \partial_{z} \omega_{z \bar{z}}+V^{\bar{z}} \partial_{\bar{z}} \omega_{z \bar{z}}=-\Lambda(z, \bar{z}) \omega_{z \bar{z}}
$$

where $\omega \equiv \omega_{z \bar{z}} \psi^{z} \psi^{\bar{z}}$. The equation (30) is easily solved by separation of variables, and the solution for the symplectic 2 -form with arbitrary separation parameter $\lambda \in \mathbb{R}$ is

$$
\omega_{z \bar{z}}^{(\lambda)}(z, \bar{z})=w_{\lambda}(z) \bar{w}_{\lambda}(\bar{z}) / V^{z} V^{\bar{z}}
$$

where

$$
w_{\lambda}(z)=\mathrm{e}^{i \lambda \int d z / V^{z}}=\left(\frac{z}{\left(1-\alpha_{1} z\right)^{A_{1}} \cdots\left(1-\alpha_{n} z\right)^{A_{n}}}\right)^{\lambda / \beta}
$$

and the constants

$$
A_{i}\left(\alpha_{1}, \ldots, \alpha_{n}\right)=\left(\alpha_{i}\right)^{n-1} \prod_{j \neq i} \frac{1}{\alpha_{i}-\alpha_{j}}
$$

are the coefficients of the partial fraction decomposition

$$
\left(V^{z}\right)^{-1}=\frac{1}{i \beta}\left(\frac{1}{z}+\sum_{i=1}^{n} \frac{A_{i}}{1-\alpha_{i} z}\right)
$$

To ensure that (31) is a single-valued function on $\mathbb{C}$, we restrict the $\alpha_{k}$ 's to all have the same phase, so that $A_{i}\left(\alpha_{1}, \ldots, \alpha_{n}\right) \in \mathbb{R}$ 's, and the parameter $\beta$ to be real-valued. The Hamiltonian equations (7) can now be integrated up with the vector field (28) and the symplectic 2-form (31), from which we find the family of Hamiltonians

$$
H_{\beta, \alpha_{i}}^{(\lambda)}(z, \bar{z})=\frac{1}{\lambda}\left(\frac{z}{\left(1-\alpha_{1} z\right)^{A_{1}} \cdots\left(1-\alpha_{n} z\right)^{A_{n}}}\right)^{\lambda / \beta}\left(\frac{\bar{z}}{\left(1-\bar{\alpha}_{1} \bar{z}\right)^{A_{1}} \cdots\left(1-\bar{\alpha}_{n} \bar{z}\right)^{A_{n}}}\right)^{\lambda / \beta}
$$

To ensure that this Hamiltonian has only non-degenerate critical points we set $\lambda=\beta$. This also guarantees that the level (constant energy) curves of this Hamiltonian coincide with the curves which are the solutions of the equations of motion (27).

Since the Hamiltonian (35) either vanishes or is infinite on its critical point set, it is easy to show that the partition function (11) is independent of $\alpha_{k}$ and coincides with the anticipated result from the Duistermaat-Heckman integration formula (2),

$$
Z(T)=\int d z d \bar{z} \omega_{z \bar{z}}^{(\beta)}(z, \bar{z}) \mathrm{e}^{i T H_{\beta, \alpha_{i}}^{(\beta)}(z, \bar{z})}=\frac{2 \pi i \beta}{T}
$$

This partition function coincides with that of the simple harmonic oscillator Hamiltonian $H=\frac{1}{\beta}\left(p^{2}+q^{2}\right) \sim z \bar{z} / \beta$. Indeed, if we set $\alpha_{i}=0$, then $\omega_{z \bar{z}}^{(\beta)}$ becomes the Darboux 2-form and $H_{\beta, 0}^{(\beta)}$ the harmonic oscillator Hamiltonian. Furthermore, the scaling function (29) vanishes, the Killing vector $V^{z}=i \beta z$ generates rotations of the plane, and the Hamiltonian flows (27) are the circular orbits $z(t)=\mathrm{e}^{i \beta\left(t-t_{0}\right)}$ about the origin in the complex plane of period $2 \pi / \beta$. 
This is the classic example of a dynamical system with WKB-exact partition function, and moreover it is the unique localizable system on a homogeneous phase space [11] (i.e. one with $\nabla \omega=0$, for which the only possible conformal motions (25) are isometries).

In fact, we can integrate up the flow equation (27) in the general case and we find that the classical trajectories $z(t)$ are determined by the equation

$$
\mathrm{e}^{i \beta\left(t-t_{0}\right)}=w_{\beta}(z(t))=\frac{z(t)}{\left(1-\alpha_{1} z(t)\right)^{A_{1}} \cdots\left(1-\alpha_{n} z(t)\right)^{A_{n}}}
$$

The coordinate change $z \rightarrow w_{\beta}(z)$ is just the finite conformal transformation generated by the vector field (28) and it maps the dynamical system $\left(\omega_{z \bar{z}}^{(\beta)}, H_{\beta, \alpha_{i}}^{(\beta)}\right)$ onto the harmonic oscillator $H \propto w \bar{w}, \omega \propto \psi^{w} \psi^{\bar{w}}$ with circular classical trajectories $w(t)=\mathrm{e}^{i \beta\left(t-t_{0}\right)}$. This transformation is in general multi-valued and has singularities at the critical points $z=1 / \alpha_{i}$ of the Hamiltonian $H_{\beta, \alpha_{i}}^{(\beta)}$. It is therefore not a diffeomorphism of the plane for $\alpha_{i} \neq 0$ and the Hamiltonian system $\left(\mathbb{R}^{2}, \omega_{z \bar{z}}^{(\beta)}, H_{\beta, \alpha_{i}}^{(\beta)}\right)$ is not globally isomorphic to the simple harmonic oscillator.

The transformations (37) are one-to-one in a neighbourhood of the origin but are oneto-many when the energy of the system is above a critical value $E_{c}$. Asymptotically, the Hamiltonian $H_{\beta, \alpha_{i}}^{(\beta)}$ tends to a finite value which is given by

$$
E_{c} \equiv \lim _{|z| \rightarrow \infty} H_{\beta, \alpha_{i}}^{(\beta)}(z, \bar{z})=\frac{1}{\beta} \prod_{k=1}^{n}\left(\alpha_{k} \bar{\alpha}_{k}\right)^{-A_{k}}
$$

If we consider the effect of the change of variable (37) on the Hamiltonian system with $H=w_{\beta} \bar{w}_{\beta}$, then circular orbits of energy less than $E_{c}$ are mapped into closed orbits which are contractible to the origin and are in a one-to-one correspondence with the domain orbits in the complex $z$-plane. As the energy tends towards $E_{c}$ these orbits grow larger and at the critical energy they actually reach infinity and return in a finite time determined by the frequency $\beta$. Above the critical energy the transformation (37) is in general one-to-many whereby single orbits are mapped to distinct orbits about each of the $n$ singular points $\left\{z_{k}=1 / \alpha_{k}\right\}_{k=1}^{n}$ of the Hamiltonian. This complicated behaviour of the conformal flows (37) is in marked contrast to the nature of the harmonic oscillator orbits which always just encircle the origin.

These unusual characteristics of the conformal flows (27) are best illustrated by explicit examples. First, we consider the case when the Hamiltonian vector field is quadratic in $z$, $V^{z}=i \beta z(1-\alpha z)$. In this case the flow equation (37) can be solved explicitly to give

$$
z(t)=\frac{1}{\alpha+\mathrm{e}^{-i \beta\left(t-t_{0}\right)}}
$$

The orbit (39) describes a circle in the complex plane centered, for total energy $\beta H=E$, at the point $E \bar{\alpha}\left(E|\alpha|^{2}-1\right)^{-1}$ and of radius $\left.\sqrt{E}|E| \alpha\right|^{2}-\left.1\right|^{-1}$ (Fig. 1). In this case the (invertible) Möbius transformation (37) effectively maps the point at $w_{\beta}=\infty$ to $z=1 / \alpha$, and the flows 


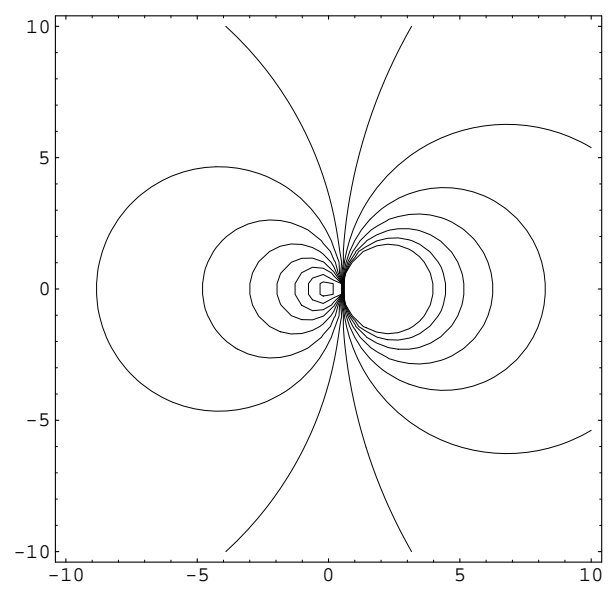

Figure 1: Conformal flows for the quadratic generator $V^{z}=i z(1-z)$.

(39) are unbounded and go out to infinity as $E \rightarrow 1 / \alpha \bar{\alpha}$. Notice that this particular example is also applicable to the compactified case where $\mathcal{M}$ is the Riemann sphere $S^{2} \simeq \mathbb{C} \cup\{\infty\}$. There the conformal group is the finite-dimensional Lie group $S L(2, \mathbb{C}) / \mathbb{Z}_{2} \simeq S O(3,1)$ of projective conformal (Möbius) transformations (i.e. for which $V^{z}(z)$ is at most quadratic in $z)$ and volume forms contain an additional factor $(1+w \bar{w})^{-2}$ associated with the compactness of $S^{2}$. In this case the Möbius transformation $z \rightarrow w_{\beta}(z)$ above is a diffeomorphism of the Riemann sphere. As for the plane, however, the conformal dynamics (39) are quite different than the isometric dynamics generated by the usual height function [6, 7, 11], and this is related to the fact that while the isometry group $S O(3)$ of $S^{2}$ is compact, its conformal group is not. The conformal group structures on spaces like $S^{2}$ give novel generalizations of the localizable systems which are usually associated with coadjoint orbits of the appropriate isometry groups and the quantization of spin systems [6, 7, 11, 12].

Next, we consider the more complicated example of the cubic conformal Killing vector $V^{z}=i \beta z\left(1-\alpha^{2} z^{2}\right)$ for which the classical trajectories are determined by the equation

$$
\mathrm{e}^{i \beta\left(t-t_{0}\right)}=\frac{z(t)}{\sqrt{1-\alpha^{2} z^{2}(t)}}
$$

Here the more complicated trajectories which coincide with the level curves of the Hamiltonian exhibit a distinct difference between the usual circular orbits of the linear (harmonic oscillator) and quadratic vector field discussed above. Additionally, in this case for energies above $E_{c}=1 / \beta|\alpha|^{2}$ we realize a one-to-two mapping (37) of the plane as the point at $w=\infty$ is now mapped to $z= \pm 1 / \alpha$. For the example $\alpha=\beta=1$ depicted in Fig. 2 there is a central hour-glass shaped region which can be seen to be in a one-to-one correspondence with the domain orbits. For energies greater than $E_{c}=1$ in this case, the classical trajectories of the system depend crucially on initial conditions as there are equivalent orbits about each 


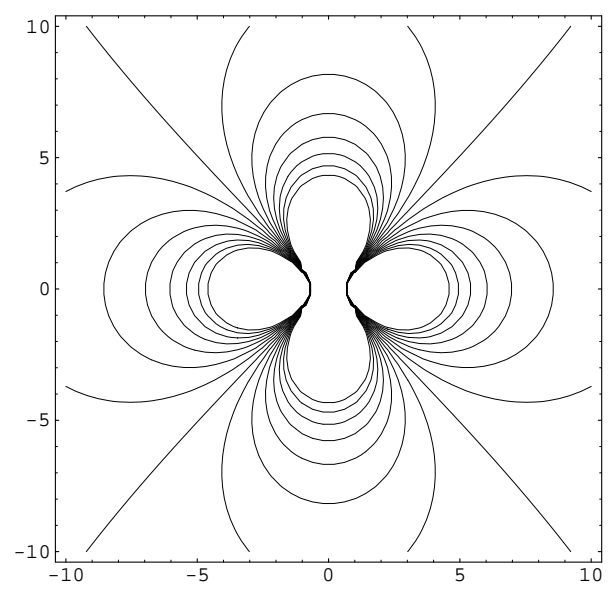

Figure 2: Conformal flows for the cubic generator $V^{z}=i z\left(1-z^{2}\right)$.

singularity at $z= \pm 1$.

The apparent equivalence between localizable Hamiltonian systems and harmonic oscillator Hamiltonians is also observed for those which generate isometries [11. It is a consequence of the fact that these Hamiltonians generate circle actions, which is the basic ingredient in the Duistermaat-Heckman theorem. This large degree of symmetry in the theory is precisely what is required to reduce the complicated integrations in (11) to Gaussian (harmonic oscillator) ones and hence render the semi-classical approximation to the partition function exact. It would be interesting to construct conformal integrable models on more general spaces other than the ones we have considered above where the equivalence with the harmonic oscillator dynamics was quite transparent because of the holomorphic-antiholomorphic decomposition of the Hamiltonian system. In the general case we expect that more complicated Hamiltonians which generate conformal motions will share common features with those which are isometry generators, but the dynamics of these systems will be quite different. These different dynamical structures could play an important role in path integral localizations which are expressed in terms of trajectories on the phase space [5, 10]. It would be very interesting to see if these general conformal symmetries of the classical theory remain unbroken by quantum corrections in a quantum mechanical path integral generalization. The absence of such a conformal anomaly could then lead to a generalization of the above extended localizations to path integral localization formulas. The appearance of the larger (non-compact) conformal group in certain settings may also lead to interesting new structures, such as in coadjoint orbit quantization [6, 7, 11, 12] or the nonabelian generalizations of the Duistermaat-Heckman theorem [8] which employ the full isometry group of the phase space.

We wish to thank D. Austin, R. Froese, I. Kogan, A. Polyakov, A. Niemi and O. Tirkkonen for helpful discussions. 


\section{References}

[1] J. J. Duistermaat and G. J. Heckman, Invent. Math. 69 (1982), 259; 72 (1983), 153

[2] M. F. Atiyah and R. Bott, Topology 23 (1984), 1

[3] N. Berline and M. Vergne, C. R. Acad. Sci. Paris 295 (1982), 539; Duke Math. J. 50 (1983), 539

[4] M. F. Atiyah, Asterisque 131 (1985), 43; J.-M. Bismut, Commun. Math. Phys. 98 (1985), 213; 103 (1986), 127; A. Hietämaki, A. Yu. Morozov, A. J. Niemi and K. Palo, Phys. Lett. B263 (1991), 417; T. Kärki and A. J. Niemi, in Proc. XXVII Intern. Ahrenshoop Symp., eds. D. Lüst and G. Weigt, DESY 94-053 (1994), 175

[5] M. Blau, E. Keski-Vakkuri and A. J. Niemi, Phys. Lett. B246 (1990), 92

[6] A. J. Niemi and P. Pasanen, Phys. Lett. B253 (1991), 349

[7] E. Keski-Vakkuri, A. J. Niemi, G. W. Semenoff and O. Tirkkonen, Phys. Rev. D44 (1991), 3899

[8] E. Witten, J. Geom. Phys. 9 (1992), 303

[9] H. M. Dykstra, J. D. Lykken and E. J. Raiten, Phys. Lett. B302 (1993), 223

[10] A. J. Niemi and O. Tirkkonen, Ann. Phys. 235 (1994), 318

[11] R. J. Szabo and G. W. Semenoff, Nucl. Phys. B421 (1994), 391

[12] G. W. Semenoff and R. J. Szabo, Mod. Phys. Lett. A9 (1994), 2705

[13] M. Blau and G. Thompson, J. Math. Phys. 36 (1995), 2192

[14] L. Hörmander, The Analysis of Linear Partial Differential Operators, Springer-Verlag (Berlin) (1983) 